University of Nebraska - Lincoln

DigitalCommons@University of Nebraska - Lincoln

Faculty Publications in Computer \& Electronics Electrical \& Computer Engineering, Department Engineering (to 2015)

2011

\title{
Power Controlled Multirate Multimedia Transmission in Self- encoded Spread Spectrum over Dynamic Fading Channels
}

\author{
Liang Chi \\ University of Nebraska-Lincoln, Ichi@unomaha.edu \\ Poomathi Duraisamy \\ University of Nebraska-Lincoln, pduarisamy@unomaha.edu \\ Won Mee Jang \\ University of Nebraska-Lincoln, wjang1@unl.edu \\ Lim Nguyen \\ University of Nebraska-Lincoln, Inguyen1@unl.edu
}

Follow this and additional works at: https://digitalcommons.unl.edu/computerelectronicfacpub

Part of the Computer Engineering Commons

Chi, Liang; Duraisamy, Poomathi; Jang, Won Mee; and Nguyen, Lim, "Power Controlled Multirate Multimedia Transmission in Self-encoded Spread Spectrum over Dynamic Fading Channels" (2011). Faculty Publications in Computer \& Electronics Engineering (to 2015). 70.

https://digitalcommons.unl.edu/computerelectronicfacpub/70

This Article is brought to you for free and open access by the Electrical \& Computer Engineering, Department of at DigitalCommons@University of Nebraska - Lincoln. It has been accepted for inclusion in Faculty Publications in Computer \& Electronics Engineering (to 2015) by an authorized administrator of DigitalCommons@University of Nebraska - Lincoln. 


\title{
Power Controlled Multirate Multimedia Transmission in Self-encoded Spread Spectrum over Dynamic Fading Channels
}

\author{
Liang Chi, Poomathi Duraisamy, Won Mee Jang and Lim Nguyen \\ Computer and Electrical Engineering \\ University of Nebraska Lincoln, \\ Omaha, NE, 68182, USA \\ lchi,pduarisamy@unomaha.edu, wjang1,lnguyen1@unl.edu
}

\begin{abstract}
In this paper, we propose to apply power control to self-encoded spread spectrum (SESS) in multirate and multimedia applications. The transmitter employs power control to to combat dynamic channel fading and the receiver employs decorrelation to reduce interference from multiple applications. We show that iterative detection can significantly improve the system performance. The proposed approach also improves the power efficiency which is essential in battery-driven communications such as mobile cellular systems.
\end{abstract}

\section{INTRODUCTION}

Multirate system has many applications in wireless communications such as speech, image and file transfer [1]. In multirate transmission, each application has its own data rate. Self-encoded spreading spectrum uses the previous $\mathrm{N}$ bits as the spreading sequence where $\mathrm{N}$ is the length of time varying spreading code. One of the advantages of this spreading scheme is that the information bit is stored in the spreading sequence itself which provide additional bit information for detection.

In section II, we present the mutilrate transmission model and propose a special extended signature vector (ESV) as spreading signature in the multirate model. We also introduce power control for dynamic flat fading. The crucial assumption for power control is that the transmitter has information about all channels between the transmitter and active receivers [2]. The fading information can also be obtained from the receiver through feedback in frequency-division duplex (FDD). The transmitter can directly perform the channel estimation in time-division duplex (TDD) mode. The receiver employs decorrelation method to reduce the cross talk between multiple applications followed by iterative detection to improve the bit-error rate (BER) performance. Section III presents the simulation results of the proposed schemes. The distribution of the power control at the transmitter and the power gain improvement are also displayed.

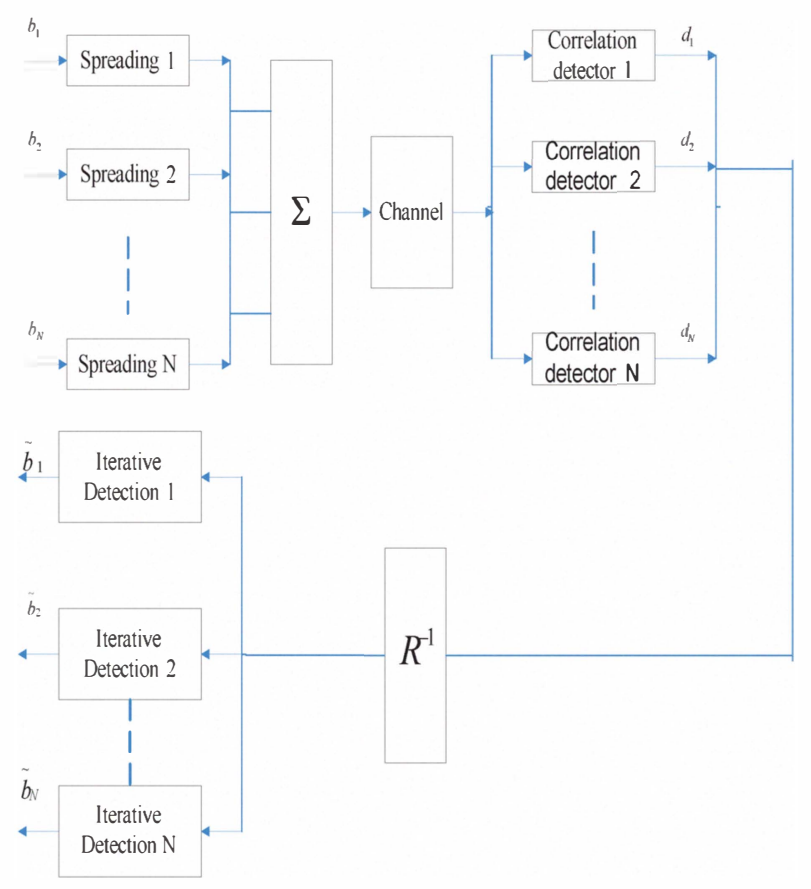

Fig. 1. Block diagram of multirate SESS.

\section{System MOdel}

Fig. 1 illustrates an example block diagram of multirate SESS where each application has it own bit rate. Since the signals of all applications are spread over the entire bandwidth, the different data rates result in different processing gains. The combined signal from all applications at the receiver undergoes decorrelation detection to remove the cross-talk among the applications. Iterative detection is then applied to improve the system performance. Finally, the training sequence is applied to reduce self-interference (SI) at a low signal-to-noise ratio (SNR). We consider three applications with different data rates. Application-1 is 64 chips/bit, Application-2 is 32 chips/bit and Application-3 is 16 chips/bit. All applications go through the same flat fading channels. 


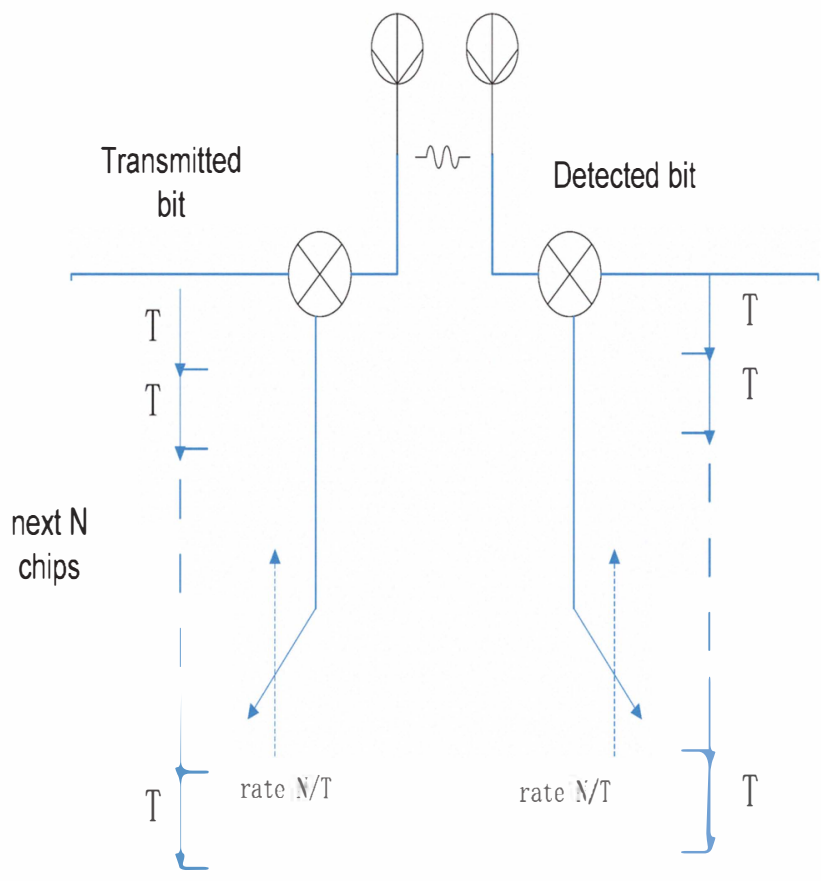

Fig. 2. SESS Transmitter and Receiver.

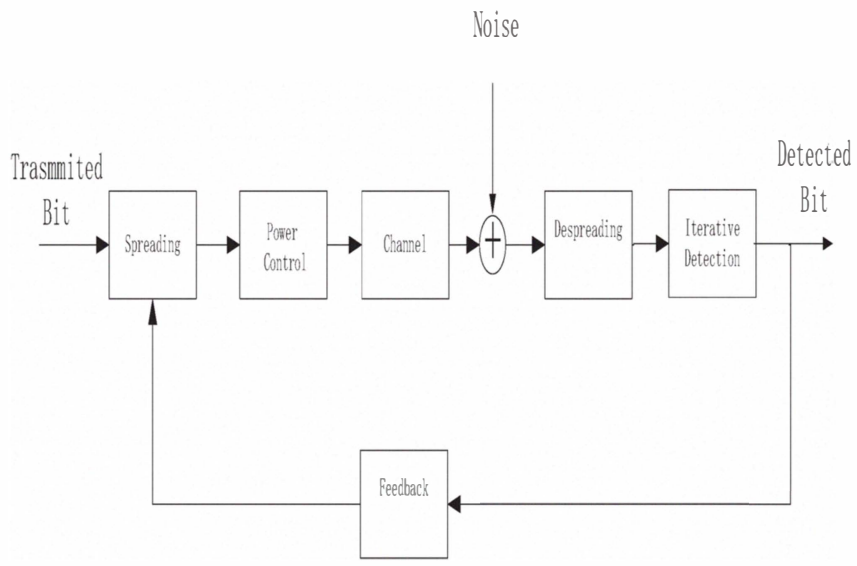

Fig. 3. Feedback detection structure for one application in the multirate communication.

Fig. 2 shows the block diagram of SESS transmitter and receiver. $\mathrm{T}$ is the bit duration, and $\mathrm{N}$ is the length of the spreading sequence. The current transmitting bit is spread by the output of the delay registers that stores the previous $\mathrm{N}$ bits. Thus, the current bit will also exist in the next $\mathrm{N}$ spreading sequences, albeit in different positions in each subsequent sequence. The receiver reverses the process to generate the despreading sequence that consists of $\mathrm{N}$ previously detected bits for dispreading the incoming bit. Fig. 3 shows the proposed feedback structure which not only can be used for synchronization but also can provide the fading channel estimation. The structure is essential for power control scheme in FDD.
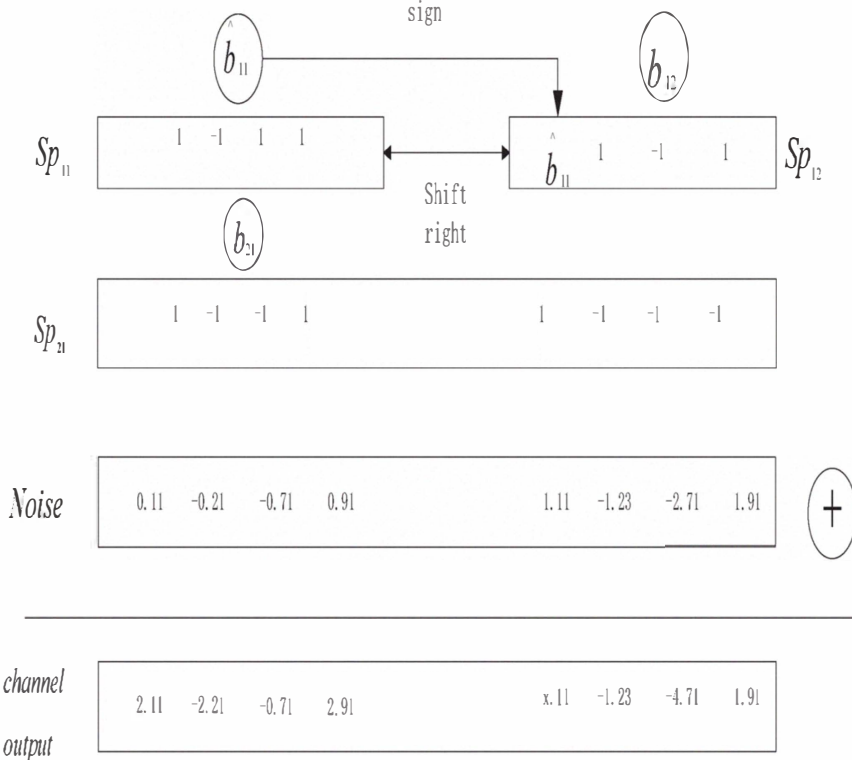

Fig. 4. Contruction of the ESV in multirate system using SESS.

Now consider a synchronous system with $\mathrm{L}$ applications sharing the same fading channel. The receiver input signal is given by

$$
r=\lambda S A b+n
$$

where $\lambda$ is the channel fading, and $S$ is the $L \times N$ matrix of signature vector. $N$ is the maximum chip length. $A$ is the amplitude of each applications, and $b$ is the vector of transmission bits. $n$ is additive white Gaussian noise with variance $\sigma^{2}$. In the multi-rate system, $S$ is different from that of the single-rate system. Rows are extended, and the overall structure is more complicated. The construction of ESV for $S$ is described below.

Fig. 4 shows two-application case in the multirate transmission. Application-1 is $N_{1}=4$ chips/bit (sp1). Application-2 is $N_{2}=8$ chips/bit (sp2). The length of the channel spreading = $\max \left(N_{1}, N_{2}\right)=8$. The number of bits in application-1 is $m_{1}=$ 2. The number of bits in application-2 is $m_{2}=1$. Therefore, the structure of ESV is

$$
S_{\left(m_{1}+m_{2}\right) \times N_{\max }}=\left(\begin{array}{cc}
s p 1 & 0_{1,4} \\
0_{1,4} & s p 1 \\
s p 2_{1} & s p 2_{2}
\end{array}\right)
$$

where $s p 2_{1}$ and $s p 2_{2}$ are the first and second half of $s p 2$. The ESV in (2) has these distinct characters: a) Any of the two sequences in the same application are orthogonal. This is obvious because different sequence in the same application takes up different time interval. Therefore, the previous sequence has no cross talk with its future sequence. b) The crosstalk of application-p's $f$ th sequence and application-q's $r$ th sequence can be easily calculated by ESV. Define $\operatorname{ESV}(x)$ as the $x$ th 
row of the ESV. Then,

$$
\rho_{p, q}(f, r)=E S V\left(\sum_{i=1}^{p-1} m_{i}^{p}+f\right) E S V\left(\sum_{j=1}^{q-1} m_{j}^{q}+r\right)^{T}
$$

where $m_{i}^{p}$ and $m_{j}^{q}$ are the $i$ th bit of application- $p$ and the $j$ th bit of application- $q$, respectively. Therefore, we can see that the structure of Eq. (2) is the signature waveform of the mulitrate transmission. The structure will be used for further detection process such as the decorrealtion and iterative detection. With the construction of ESV completed, we now proceed with multirate system model that the random flat fading $\lambda$ has the variance $\sigma_{\lambda}^{2}$ with the uniform distribution at $[0.1,1.9]$. Therefore,

$$
X \in[0.1,1.9] \text { and } E(X)=1 .
$$

Assuming that transmitter power control

$$
r=(1 / \sqrt{X}) \times \lambda S A b+n
$$

where the power enhancement due to the power control is

$$
Y=1 / X
$$

Then, the detected bit can be obtained as

$$
\tilde{b}_{k}=\operatorname{sign}\left(x_{k}(r)\right)
$$

$x_{k}(r)$ is the despreaded value with the $k$-th spreading sequence. We try to minimize the mean square error,

$$
J_{\min }=\|S x-r\|^{2}
$$

where $\|\cdot\|$ is the Euclidian norm . The solution to Eq. (8) is

$$
x(r)=S^{+} r
$$

where the $K \times L$ matrix $S^{+}$is the Moore-Penrose generalized inverse of $S$ [3]. In general, $L<K$ and $R$ is the correlation matrix. Therefore,

$$
S^{+}=S^{T}\left(S S^{T}\right)^{(-1)} \text { and } R=\left(S S^{T}\right)^{-1} .
$$

\section{NUMERICAL RESULTS}

\section{A. Correlation Detection}

In this section, we present the numerical results for the power control. We focus on the distribution of the power as well as the BER performance. Fig. 5 illustrates the distribution of the power control values during the transmission. The histogram shows that all the values concentrate between 0.52 to 10 . The dense interval is $[0,1]$ where the power control values up to $4.2 \times 10^{6}$ account for $66.98 \%$ of all samples. The mean value and variance are calculated as follow

$$
\begin{array}{r}
E(Y)=1.6386=4.9(d B), \\
\operatorname{Variance}(\mathrm{Y})=2.6049=9.5(d B) \\
\text { and } \operatorname{peak}(Y)=9.9021
\end{array}
$$

where $Y$ is the power control value in Eq. (6).

Fig. 6 shows the multirate SESS performance comparison power control in dynamic fading channel without iterative detection. All three applications go through the channel with the

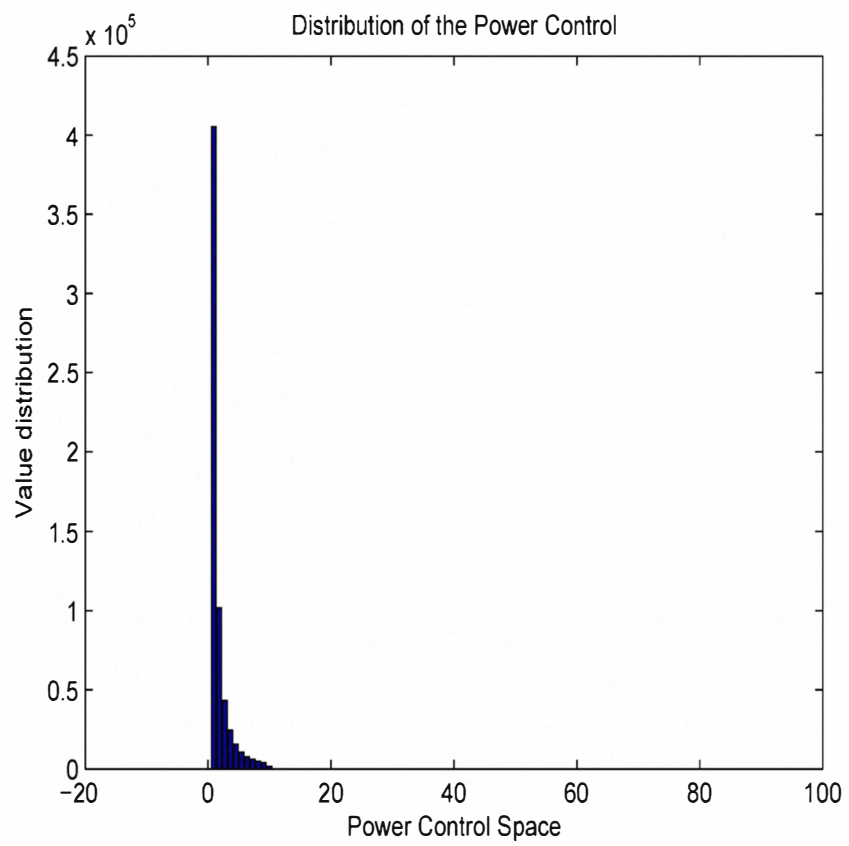

Fig. 5. Distribution of the value of power control, 1,6275460 samples.

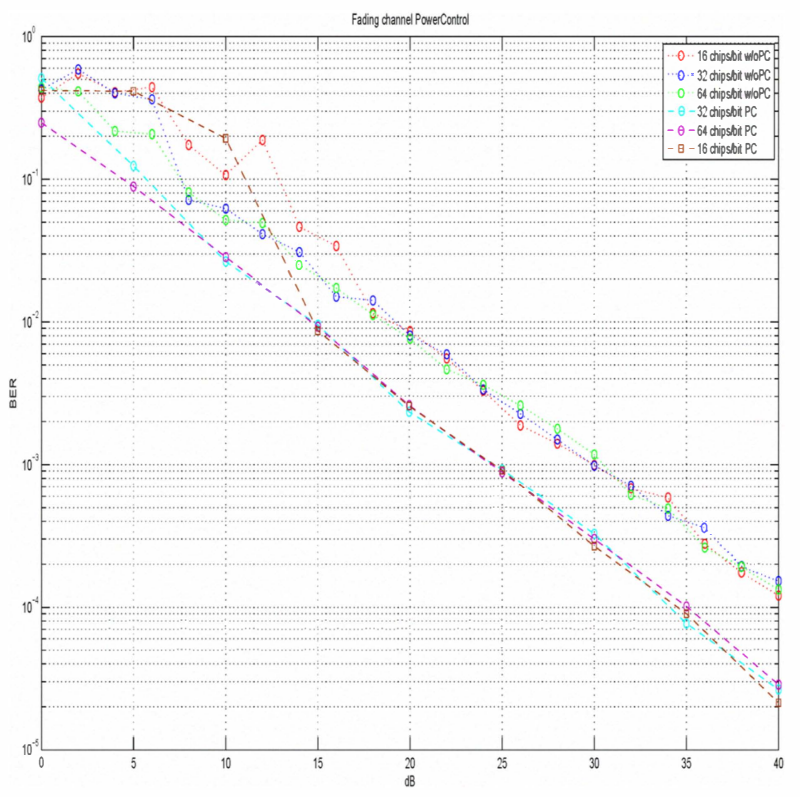

Fig. 6. Power control in the multirate transmission, dynamic fading channel, without iterative detection.

random flat fading. They exhibit nearly the same performance although they have different processing gain. Power control achieves a $5 \mathrm{~dB}$ gain. In both cases, we observe SI is high at a low SNR. 


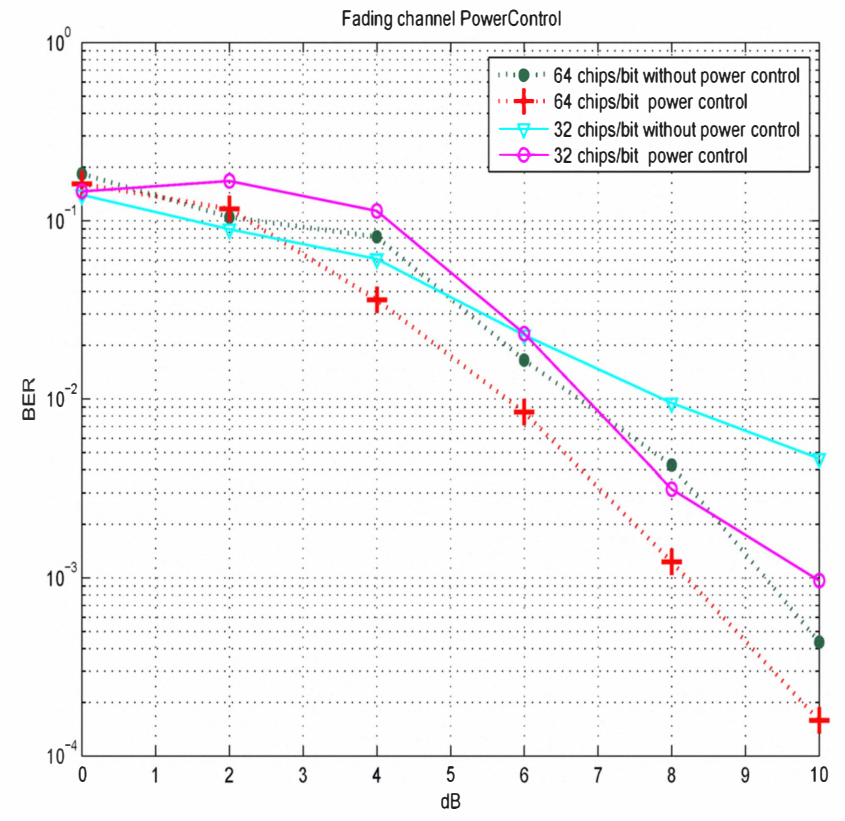

Fig. 7. Power control in the multirate transmission, dynamic fading channel, iterative detection.

\section{B. Iterative Detection in the Multirate Communication System}

Fig. 2 shows that the information bit itself is employed in the spreading sequence which then can provide time diversity to employ the detection. This iterative detection improves the BER performance significantly over the fading channel [4]. Even in the multirate communication system, the iterative detection can still be employed for each application. However, the iterative deteciton in multirate system require that we separate the combined received signal sequence for each application. To achieve this goal, we employ both decorrelation technique and interference cancelation. With interference cancelation, we estimate the despreading sequences and detected bits of other applications from the decorrelation detection. We then subtract the combined estimated spreading signals of the remaining applications from the received signals in order to cancel the interference to the desired application, which can then be further recovered with iterative detection.

Fig.7 shows the multirate SESS performance power control for dynamic fading channel with iterative detection. It is clear that power control improves about $2 \mathrm{~dB}$ in both applications of $64 \mathrm{chips} / \mathrm{bit}$ rate and $32 \mathrm{chips} / \mathrm{bit}$. Comparing to Fig. 6, we observe that the iterative detection achieves $10^{-3}$ BER at $E_{b} / N_{o}=8 \mathrm{~dB}$ while decorrelation only (without iterative detection) $20 \mathrm{~dB}$ with $12 \mathrm{~dB}$ SNR improvement. It is obvious that iterative detection can improve the multirate system performance significantly.

\section{Feedback Detection and Training sequence}

Fig. 7 also shows that the effect of self interference can be worsen with iterative detection, especially at low SNR where the estimates are simply to unreliable. To mitigating this effect

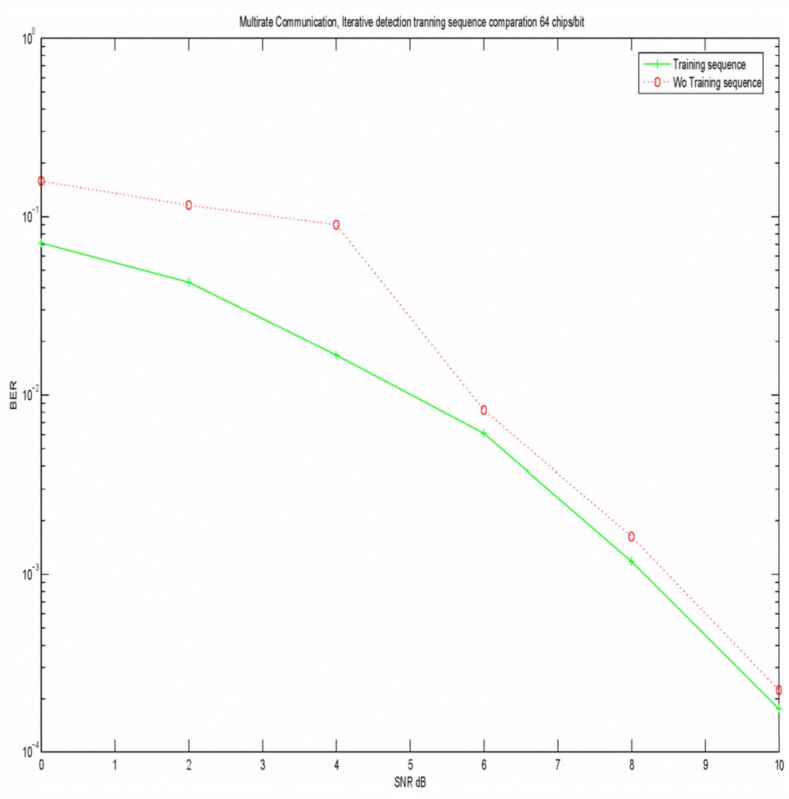

Fig. 8. Training sequence for the multirate transmission in dynamic fading channel, iterative detection, $\mathrm{N}=64$ chips/bit.

we propose to employ training sequences which are typically used for synchronization and channel estimation.

In our study, the training sequence is generated every $10 \mathrm{~N}$ bits periodically to adjust the received signal sequence. Once the synchronization has been achieved, the transmitter continue to send the signal. For the next $10 \mathrm{~N}$ bits, the synchronization process will repeat until the last bit is received. We consider a two-way training sequence. If Base Station (BS) receives erroneous spreading sequence from Mobile Station (MS), BS updates its spreading sequence with the received sequence to ameliorate the SI effect at MS.

Fig. 8 shows the example comparison for training sequence with the iterative detection In the figure, we can see clearly the advantage the training sequence especially at a low SNR. From $1 \mathrm{~dB}$ to $4 \mathrm{~dB}$, the performance with training sequence can achieve $10^{-2}$ BER However, without training sequence, the performance just diplays $10^{-1}$ BER only. Even at a high SNR, the training sequence still displays the a little higher performance, about $0.2 \mathrm{~dB}$. Similar results has been obtained for $\mathrm{N}=16$ and 32 .

\section{CONCLUSION}

In this paper, we present the mutltirate SESS over dynamic fading channels. At the transmitter, power control is employed to combat the channel fading dynamics. Meanwhile, at the receiver, decorrelation and iterative detection improve the system performance by $12 \mathrm{~dB}$ SNR. The training sequence can make the performance better at lower SNR reducing the self-interference effect. From the simulation result, we can see that the power control can achieve an additional $2 \mathrm{~dB}$ gain over without power control in dynamic fading channels. Therefore, 
our proposed system exhibits at least $14 \mathrm{~dB}$ SNR gain over conventional multirate systems.

\section{ACKNOWLEDGMENT}

This work was supported by the contract award FA955008-1-0393 from the Air Force Office of Scientific Research.

\section{REFERENCES}

[1] P. P. Vaidyanathan, "Multirate digital filters, filter banks, polyphase networks, and applications: a tutorial," Proceedings of IEEE, vol.78, no.1, Jan. 1990.

[2] B. R. Vojcic and W. M. Jang, "Transmitter precoding in synchronous multiuser communications," IEEE Trans. Commun., vol. 46, No. 10, pp. 1346-1355, Oct. 1998.

[3] S. Verdu, Multiuser detection, pp. 234-244, Combridge Press, US, 1998

[4] Y. S. Kim, W. M. Jang, Y. Kong and L. Nguyen, "Chip-interleaved self-encoded multiple access with iterative detection in fading channels," Journal of Communications and Networks, vol. 9, no. 1, pp. 50-55, Mar. 2007.

[5] T. H. Chang, W. C. Chiang, Y. W. Peter Hong and Chong-Yung Chi, "Training sequence design for discriminatory channel estimation in wireless MIMO systems," IEEE Trans. Proc., vol. 58, no. 12, Dec. 2010. 